\title{
La Face Cachée de la Décentralisation : Réseaux, Clientèles et Capital Social
}

René Lemarchand

\section{Q OpenEdition}

\section{Journals}

Édition électronique

URL : http://journals.openedition.org/apad/522

DOI : 10.4000/apad.522

ISSN : 1950-6929

Éditeur

LIT Verlag

Édition imprimée

Date de publication : 1 décembre 1998

\section{Référence électronique}

René Lemarchand, «La Face Cachée de la Décentralisation : Réseaux, Clientèles et Capital Social », Bulletin de l'APAD [En ligne], 16 | 1998, mis en ligne le 06 octobre 2006, consulté le 07 septembre 2020. URL : http://journals.openedition.org/apad/522 ; DOI : https://doi.org/10.4000/apad.522

Ce document a été généré automatiquement le 7 septembre 2020

Bulletin de l'APAD 


\title{
La Face Cachée de la Décentralisation : Réseaux, Clientèles et Capital Social
}

\author{
René Lemarchand
}

1 "Parler de décentralisation aujourd'hui est une gageure parce qu'elle déclenche, tout comme la décolonisation à une autre époque, des passions d'une telle violence, qu'on réalise seulement à ce moment les véritables enjeux qu'elle recouvre" (Cauris, Bamako, décembre 1994).

2 Instruit de cette mise en garde, c'est avec prudence que je m'aventure sur ce terrain semé d'embûches que représente la décentralisation. Si les enjeux sont à la mesure des pussions qu'ils suscitent, il en est un au moins sur lequel l'accord est quasi-unanime : l'enjeu central de la décentralisation, c'est la démocratie à la base. Mais ce qui pour certains est une vérité d'évidence - il n'y a pas de démocratie véritable sans une participation active des citoyens de base à l'élaboration des décisions qui les concernent - revêt beaucoup d'ambiguïté dès lors qu'on s'interroge sur les moyens à mettre en œuvre pour y parvenir. C'est ici que les controverses - et les passions surgissent.

3 C'est en vain qu'on chercherait un modèle uniforme, une sorte de "one size fits all", applicable à toute communauté indépendamment du profil qui la caractérise. Ce qui intéresse le chercheur en sciences sociales c'est, non seulement l'historicité des sociétés africaines, les mutations introduites par l'économie, l'idéologie, la religion, mais aussi la manière dont celles-ci sont perçues, et vécues, par la base. Or, force est de constater que les développeurs et bailleurs de fonds ont été extraordinairement peu attentifs à ces dimensions, ceux-ci s'intéressant davantage aux critères de "faisabilité", de durabilité et de rentabilité, définis selon leurs propres conceptions de la bonne gouvernance.

4 Comment, dans ces conditions, faire sortir les projets de décentralisation de la gangue technocratique qui les enrobe ? Comment "opérationnaliser" la recherche dans le sens d'une meilleure synergie entre chercheurs et développeurs? Où situer le "terrain 
favorable" à un échange fécond entre les uns et les autres? Telles sont, grosso modo, les questions autour desquelles nous voudrions orienter les débats.

Pour mieux délimiter le champ de ma réflexion, permettez-moi d'abattre mes cartes : pour reprendre un thème cher à Robert Putnam (1993), la décentralisation est avant tout, me semble-t-il, affaire de capital social, c'est-à-dire de ressourcement à la base ; c'est par la mise en place de réseaux de solidarités horizontales, fondées sur la confiance mutuelle, que doit s'étayer la vie communautaire dans le sens d'une participation plus active aux affaires des collectivités de base. Ceci vaut aussi bien pour l'Afrique que pour les États du Nord. Or cette action, pour être efficace, doit tenir compte, et éventuellement se substituer au jeu des multiples solidarités de type vertical qui, partout en Afrique, sous une forme ou une autre, limitent la participation, détournent les financements, entravent l'allocation des ressources, et renforcent l'emprise de l'État. En bref, comment opérer le passage du clientélisme politique au capital social ? Nous y reviendrons dans un moment.

6 Ma démarche s'inspire de ma double identité professionnelle - en partie de mes récents états de service à Abidjan et Accra en tant que consultant auprès de l'USAID pour la gouvernance et la démocratie, et en partie de mes travaux antérieurs sur le clientélisme politique. J'ajoute à cela que ma réflexion a également profité des très riches travaux de l'APAD, dans le domaine de la dynamique des pouvoirs locaux. Je pense notamment aux travaux de Roch Mongbo sur le Bénin, de Bréhima Kassibo et Bréhima Béridogo sur le Mali, de Mariatou Koné sur la Côte d'Ivoire, de Pierre-Joseph Laurent et Jean-Pierre Jacob sur le Burkina, de Giorgio Blundo, Thomas Bierschenk et Jean-Pierre Olivier de Sardan sur les phénomènes de courtage, de Philippe Lavigne Delville, "agent double", suivant ses propres termes, et doublement éclairant sur les rapports entre sciences sociales et développement.

7 Il n'est pas question, dans le cadre de cette discussion, d'inventorier les kyrielles de projets mis en chantier par les coopérants français, allemands, canadiens ou américains -encore moins d'arpenter les arènes qui ont présidé à leurs avatars. Notre ambition est plus modeste, malgré son esprit quelque peu destructeur - lancer quelques coups de boutoir contre les cloisons qui séparent les approches des bailleurs de fonds de celles des sciences sociales (auxquelles s'ajoutent les obstacles que constituent parfois les autorités chargées de la mise en place des projets de décentralisation), et explorer les voies d'une cohabitation plus fructueuse.

D'évidence, la face cachée de la décentralisation c'est précisément celle que masque ce cloisonnement - celle que s'efforce de mettre en lumière les sciences sociales et que mettent entre parenthèses les développeurs. C'est celle de la "politique par le bas", celle des clientèles rurales, des réseaux confrériques, des autorités villageoises, des coopératives rurales, des courtiers et intermédiaires. C'est aussi celle des solidarités horizontales qui se tissent à travers l'émergence de nouvelles associations, souvent en concurrence ou en porte-à-faux avec les big men issus, les uns du pouvoir central, les autres de la tradition, parfois de la rencontre des deux.

Chercheurs et développeurs : le fossé épistémologique

9 Avant d'aller plus avant, il convient de noter le flou conceptuel qui entoure les enjeux de la décentralisation. Celle-ci évoque des réalités fort différentes suivant le contenu que lui donnent les développeurs, les autorités gouvernementales ou les chercheurs en sciences sociales : déconcentration administrative, renforcement de la société civile, augmentation des capacités institutionnelles, mise en place de conseils communaux et 
régionaux, le plus souvent sous l'œil vigilant de commissions d'experts, reconnaissance accrue de l'autorité de la chefferie, émergence de courtiers du développement, autant de manières différentes de concevoir les enjeux de la décentralisation.

10 La confusion qui en résulte n'est pas le fait du hasard. Développeurs, autorités gouvernementales, et chercheurs ont chacun leur façon de voir les choses. Dans bien des cas, les premiers s'inspirent de schémas institutionnels empruntés à leurs pays d'origine (les scandinaves ne jurent que par l'ombudsman, les américains par la société civile, les français par l'élargissement des compétences communales, etc.); les secondes, tout en chantant les louanges de la démocratie à la base, n'ont de cesse que d'affermir l'autorité des représentants de l'Etat face au pouvoir local.

Quant aux chercheurs, le moins qu'on puisse dire est que leur vision de la décentralisation s'accommode d'une très large gamme de priorités, allant de la société civile aux capacités institutionnelles, de l'analyse des terroirs à l'intervention des courtiers du développement.

12 Sans doute, cette diversité d'approches n'est-elle pas étrangère aux rapports de dépendance qui parfois relient les chercheurs aux bailleurs de fonds. Le mercenariat académique est une réalité incontournable. Tout aussi évident, cependant, est le fossé épistémologique qui sépare ce qu'Olivier de Sardan appelle les logiques de la connaissance et les logiques de l'action: "La connaissance et l'action mobilisent des registres de légitimation quasi incommensurables (...) L'une se garde de croire, l'autre en a besoin. L'une se veut désintéressée, l'autre revendique d'avoir les mains dans le cambouis. L'une exige du temps, l'autre n'en a pas. L'une veut observer les processus sociaux, l'autre veut les orienter ou les contrôler" (1995:190).

13 À logiques différentes, langages différents. Celui des développeurs, de l'USAID en particulier, s'émaille d'une foultitude de sigles et d'abréviations que seuls les initiés peuvent décrypter, de formules qui occultent plus qu'elles n'éclairent, de cadres conceptuels qui n'entretiennent qu'un rapport lointain avec les réalités du monde rural.

ercheurs, de leur côté, ne sont pas toujours exempts d'un penchant pour les formulations absconces. Encore ne faut-il pas confondre les abus de jargon avec les exigences d'une pensée qui s'efforce de traduire la complexité des phénomènes observés.

15 Alors que les sciences sociales se préoccupent de plus en plus des questions d'ordre politique - notamment des "nouveaux langages du politique", pour reprendre le titre de l'ouvrage de Denis-Constant Martin (1998) - les développeurs, à de rares exceptions, tendent à évacuer la politique. Dans cet univers plus ou moins figé, aseptisé, la problématique de la décentralisation est réduite à des approches essentiellement technocratiques, fondées sur l'accès aux technologies de l'information par le biais de connexions internet, les séminaires de formation, les schémas du "logframe" (logical framework), et l'appui à une société civile plus ou moins homogène, toujours porteuse de promesses et à jamais libérée des contraintes du pouvoir.

Le titre de l'ouvrage de James Ferguson - The Anti-Politics Machine (1994) - résume admirablement les tares d'un discours développementaliste, en l'occurrence celui de l'USAID, lequel, en raison même de son apolitisme, a pour effet d'occulter les véritables problèmes du monde rural - pauvreté, marginalisation, oppression - en laissant le champ libre à l'arbitraire bureaucratique. Ce que Ferguson observait au Lesotho dans 
les années 1980, James Thompson et Cheiban Coulibali le redécouvrent une décennie plus tard au Mali : "Dans plusieurs projets financés à l'extérieur, la participation des populations a consisté à effectuer des travaux définis par les agents de l'Etat et les représentants des bailleurs de fonds. Il n'était pas question que les populations participent aux prises de décision quant au choix, à la mise en œuvre et au contrôle des politiques de développement. Il était encore moins question que ces populations puissent prendre librement, et seules, certaines décisions concernant l'organisation de leur vie" (Thompson \& Ferguson 1994 : 3).

Reconnaître les attentes des communautés de base est une chose; leur donner les moyens de s'exprimer en est une autre. Comment répondre à ce défi ? Avant d'esquisser quelques pistes pour les sciences sociales, un bref retour en arrière s'impose, ne fût-ce que pour souligner les contraintes nées de l'Etat colonial. Celles-ci, en effet, ont survécu aux indépendances à travers la mise en place d'un modèle néo-patrimonial qui a réorganisé les entraves du passé plus qu'il ne les a éliminées.

Du despotisme décentralisé au clientélisme politique

18 Contrairement à l'image béni ne que ses géniteurs en ont donné, l'indirect rule a présidé à l'institutionnalisation d'un pouvoir local exonéré des mécanismes de contrôle qui autrefois assuraient sa légitimité. Autour de ce "despotisme décentralisé", pour reprendre l'expression de Mahmood Mamdani (1996), se sont cristallisées toutes sortes de pratiques et prérogatives plus ou moins arbitraires et coercitives, chacune investie par l'autorité coloniale d'une légitimité parfaitement factice, comme pour "normaliser" l'usage de la force. Ainsi s'élabora un système de native authorities, chacune, nous dit Mamdani, "armée d'un fouet et protégée par le halo de la coutume" (Mamdani 1996: 44). Notons en passant que malgré les différences formelles qui séparent l'administration directe de l'indirecte, le régime des chefs de canton de celui des native authorities, l'attitude des commandants de cercle des district commissioners, ce mode de gouvernement n'est pas l'apanage de l'Afrique anglophone. On le retrouve également, sous des formes plus centralisées mais non moins contraignantes, en Afrique francophone.

Loin de l'éliminer, les indépendances ont réaménagé ce système dans le cadre d'un clientélisme d'Etat qui a engendré une plus grande instrumentalisation des relations de dépendance sans pour autant diminuer l'arbitraire des élites locales. En témoigne l'émergence du modèle néo-patrimonial (Médard 1982), ce monstre hybride, au sein duquel s'entremêlent public et privé, personnalisme et autoritarisme, carottes et bâtons. L'introduction du vote, la mise en place de partis uniques ou dominants, la nécessité de mieux contrôler la brousse pour la mobiliser, sont autant d'additifs qui ont transformé les despotismes décentralisés en clientélismes politiques destinés à créer des courroies de transmission entre le centre et la périphérie. Si les ressources allouées aux communautés locales sont la contrepartie du soutien politique exigé par l'Etat, c'est avec la plus grande sélectivité que celles-ci sont redistribuées à l'échelon du village ou de la région. Le patronage local reproduit à ces niveaux toutes les tares du clientélisme d'Etat : favoritisme, népotisme, corruption, factionalisme, élimination des opposants.

$\mathrm{Au}$ despotisme décentralisé de l'époque coloniale se substitue un jeu politique dont la complexité - et l'âpreté - est à la mesure des rivalités entre les clientèles locales, celles-ci n'étant le plus souvent que le reflet de l'importance des enjeux fonciers et de la compétition pour l'accès aux ressources de l'Etat. L'échange, dans ces conditions, ne 
fonctionne plus selon un code social traditionnel mais suivant les affiliations aux réseaux issus du centre. C'est précisément à ce système néo-patrimonial que devaient s'attaquer les politiques d'ajustement structurel, en brisant les monopoles d'Etat, en promouvant la privatisation et la bonne gouvernance. On sait désormais que les réformes ont produit des résultats très divers, et rarement conformes aux attentes de leurs partisans. Contentons-nous de noter que les affleurements du clientélisme politique sont encore très présents, comme en témoigne, en particulier, le phénomène des "courtiers du développement", brillamment analysé par Giorgio Blundo (1995).

Contraction des réseaux clientélistes et réinvention du pouvoir local

Qui dit ajustement structurel dit démantèlement des entreprises publiques, contraction des dépenses sociales, dégraissage de la fonction publique, et appui au secteur privé. Dans l'optique qui nous concerne, c'est l'atrophie des réseaux clientélistes qui mérite d'être soulignée, en même temps que l'encouragement des bailleurs de fonds à la privatisation et à la décentralisation en tant que pôle de bonne gouvernance. Ce concept assez flou - qui évoque la transparence, la participation et l'utilisation rationnelle des ressources de l'Etat, c'est-à-dire leur privatisation - sonne le glas de l'Etat interventionniste et le dépérissement de ses clientèles rurales. Alors que l'Etat néo-patrimonial entretient la confusion entre le public et le privé, l'ajustement structurel vise à les séparer. Corollaire de ce cloisonnement, la société civile est alors perçue comme l'instance privilégiée à travers laquelle s'installent les contre-pouvoirs destinés à limiter l'emprise de l'Etat.

C'est ainsi qu'on assiste à partir des années 1990 à un effort soutenu des bailleurs de fonds pour promouvoir la décentralisation. Celle-ci figure au premier rang des priorités de l'USAID : au sein du Global Bureau de l'USAID/Washington est organisée une commission chargée de la gouvernance locale (Democratic Local Governance Task Force) tandis qu'au Ghana, au Mali, au Niger, au Bénin, en Tanzanie s'élaborent des programmes d'assistance aux collectivités locales. Les Canadiens, les Allemands et les Scandinaves emboîtent le pas. La France s'engage dans la voie de la coopération décentralisée, et organise de multiples partenariats entre communes métropolitaines et africaines. Partout, d'une façon ou d'une autre, l'objectif est de mettre en chantier des projets dans lesquels les populations locales se prennent en charge elles-mêmes.

À ces multiples projets de décentralisation, nombre d'Etats ont répondu par des réformes allant dans le sens d'une déconcentration du pouvoir exécutif. Dès 1972 le Sénégal avait introduit une réforme ayant pour but, comme le disait Senghor, "d'étendre les effets de la décolonisation aux populations rurales" (Jacob \& Blundo 1997: 51). Au Bénin, en 1993, les Etats Généraux de l'Administration Territoriale amorcent un processus de décentralisation visant à la constitution de nouvelles communes dotées d'une large autonomie dans l'élaboration et la gestion de leurs budgets. La même année le Mali met en place une Mission de Décentralisation dont les travaux débouchent sur une vaste réorganisation territoriale. C'est également en 1993 que le Burkina Faso crée la Commission Nationale de Décentralisation. Après de longs débats naît en 1996 un timide projet de décentralisation communale : la commune y est conçue comme "lieu d'apprentissage de la gestion des affaires publiques" (Jacob \& Blundo 1997 : 41), tout en restant sous la tutelle de l'Etat.

Il n'entre pas dans nos intentions de mesurer l'écart qui sépare l'esprit de ces réformes de leur réalité (ou irréalité). Le moins qu'on puisse dire est que l'optimisme généralement affiché par les milieux officiels tranche singulièrement avec le ton 
beaucoup plus sobre des évaluations faites par les observateurs nationaux et internationaux. Au mieux, il s'agit d'un exercice de déconcentration plutôt que d'une véritable décentralisation; au pire, dans certains cas précis (Burkina, Mali), et sous bénéfice d'inventaire, c'est à un constat d'échec qu'il faut se rendre.

Ce qui est loin d'être un échec par contre, c'est la floraison d'associations villageoises qui depuis quelques années peuplent le paysage de l'Afrique rurale. Le retrait partiel de l'Etat-patron des secteurs ruraux, les nouvelles "lucarnes d'opportunité" ouvertes par les bailleurs de fonds, la mise en disponibilité de certains agents de l'Etat dans le sillage de l'ajustement structurel, autant de facteurs qui ont stimulé l'éveil de nouveaux réseaux associatifs. Qu'il s'agisse de coopératives agricoles, d'associations de femmes, de communautés chrétiennes de base, de mutuelles, de banques populaires. d'associations de crédit, le principe de base reste le même : permettre aux collectivités rurales de se prendre en charge en mobilisant les énergies et les ressources locales. À travers ces multiples initiatives s'affirme une société civile qui devance l'Etat dans ses velléités d'autonomie. On comprend pourquoi, dans l'optique des bailleurs de fonds, l'aide à la société civile soit souvent perçue comme le chemin le plus court vers la gouvernance décentralisée.

De là à en conclure à une Afrique "tocquevillienne", où la démocratie serait inscrite dans le penchant associatif de la société civile, il n'y a qu'un pas, trop rapidement franchi par certains bailleurs de fonds. La réalité, on s'en doute, est plus complexe. L'Afrique des ONG exprime des forces contradictoires: une volonté de se prendre en charge, mais aussi une certaine incapacité à transcender l'espace de la petite communauté, de l'ethnie, du clan, du terroir. En résultent un extrême morcellement du tissu associatif, en même temps qu'un déficit de ce que Pierre-Joseph Laurent appelle "l'entente". L'entente, nous dit-il, c'est "l'élaboration d'une association capable de transcender les identités lignagères traditionnelles et de quartier", c'est "l'invention d'un nouveau lien social au village, beaucoup plus basé sur l'individu et la garantie de sa liberté d'action, et qui ne correspond plus uniquement à l'habituel «entre-sol» villageois" (Laurent 1995 : 93, 95).

L'entente c'est aussi un aspect central de cette notion de capital social analysée par Robert Putnam dans le contexte de l'Italie du nord: l'entente civique au sein des communautés lombardes et piémontaises, si différentes par l'histoire et la culture politique de l'autoritarisme du Mezzogiorno, est en effet indissociable de la capacité de leurs citoyens, dès la Renaissance, à accumuler un capital de confiance, à l'investir dans des réseaux de solidarités horizontales, et à en repartir équitablement les bénéfices.

Comment expliquer ce déficit de capital social dans le cas des sociétés africaines?

L'explication la plus évidente a trait au contexte social des communautés rurales. Le village est un champ politique complexe, traversé d'influences et d'intérêts divers, où se côtoient le monde de l'invisible et du visible, les sorciers et les guérisseurs, les gens du pouvoir et les gens de la terre, les autochtones et les allogènes, les éleveurs et les cultivateurs. C'est dans ce contexte multiforme et potentiellement conflictuel que se situe la problématique des mouvements associatifs, et que doivent se comprendre les stratégies d'évitement, de négociation, d'innovation par lesquelles ils s'affirment sur la scène politique locale. On ne saurait trop insister sur les multiples obstacles que pose cette Afrique plurielle à l'accumulation du capital social. 

association paysanne sénégalaise : "Quand un projet vient s'installer dans la zone et que les toubabs arrivent avec les financements, il y a toujours des gens qui se proposent comme représentants des villageois, et qui sont de ce fait investis pour la suite des activités comme responsables locaux du projet... Mais il faut faire attention: très souvent ces individus poursuivent exclusivement leurs intérêts personnels. Ils sont comme des mouches sur un morceau de sucre : les premières à s'y poser, mais après leur passage le sucre est irrémédiablement contaminé" (Blundo 1995: 73). Ce qui transparaît à travers les fonctions de courtage (brokerage) et de médiation exercées par ces nouveaux acteurs, c'est une sorte de clientélisme associatif, où le courtier use de ses compétences pour extraire des ressources auprès des bailleurs de fonds pour ensuite les réinvestir de façon sélective, dans le but de se constituer une clientèle rurale tout en s'appropriant au passage les bénéfices de sa médiation. Ainsi s'effrite dans le jeu des réciprocités particularistes et des détournements, le capital social dont aurait pu jouir l'association si de véritables solidarités horizontales s'étaient forgées entre ses membres.

31 Une troisième explication, qu'illustre en partie la précédente, se rapporte au rôle des bailleurs de fonds dans ce processus d'appropriation sélective. Si les phénomènes de récupération analysés par Blundo échappent le plus souvent à l'attention des intervenants c'est que leurs projets pêchent trop souvent par excès d'optimisme. Tout projet est une lecture de la réalité africaine, mais une lecture qui tend a évacuer les incertitudes. Notons à ce propos que malgré la vogue que connut en son temps l'ouvrage de Robert Putnam auprès du public anglo-saxon, la notion de capital social, et les incertitudes qui s'y rattachent, n'ont guère suscité d'intérêt auprès des experts en gouvernance de l'USAID. C'est à leur insu que la manne du développement est parfois absorbée par les "courtiers'" et leurs clientèles. Même si les bailleurs avaient les moyens d'y remédier, en particulier les "staff resources'" nécessaires au suivi des projets, il n'est pas certain que le capital social figure sur la liste de leurs priorités. Ce sont non seulement les logiques paysannes qui échappent à leur compréhension; c'est aussi l'optique des sciences sociales. Le problème de la communication (ou de l'incommunicabilité) se pose à la fois vis-à-vis des bénéficiaires et au niveau du dialogue entre chercheurs et développeurs.

Que faire?

Ceci nous amène, en guise de conclusion, à reprendre certains des thèmes abordés par Philippe Lavigne Delville dans son analyse de la place des sciences sociales dans les projets de développement (1997). À la question soulevée dans le titre de son article - "à quoi servent les sciences sociales dans les projets de développement rural ?" - nous proposons quelques réponses qui s'inspirent de notre expérience de consultant au Ghana. Le chercheur en sciences sociales a pour première obligation de débroussailler le paysage rural pour le rendre intelligible aux intervenants. Les critères de sélectivité et de pertinence sont ici essentiels. Plutôt que de se livrer à une analyse exhaustive de tout ce qui se rapporte de près ou de loin au site du projet, son rôle est de cibler ses objectifs et de repérer les variables les plus sensibles à sa réussite. C'est souvent au détriment d'un ciblage approprié que s'effectuent les études de terrain à caractère 
sociologique. Un exemple nous vient à l'esprit: dans le cadre du programme d'assistance de l'USAID à la société civile du Ghana, une équipe du National Democratic Institute (NDI) arriva un beau jour sur le terrain, armée d'un impressionnant outillage méthodologique. Au cours de nos entretiens je soulignai la nécessité de définir la société civile ; j'insistai en particulier sur l'immense diversité de ses composantes, et sur la nécessité d'arriver à un échantillonnage des groupes les plus qualifiés pour recevoir l'appui de l'USAID. L'équipe n'en fit qu'à sa guise. Résultat de l'enquête préliminaire: un rapport d'une soixantaine de pages, la moitié consacrée à un recensement des travaux académiques sur la société civile, et couronnée de deux conclusions "majeures" : (a) les femmes du Ghana ne s'intéressent pas à la politique ; (b) les populations du nord, comme les femmes, sont apolitiques. Coût de l'opération : \$ 76.000

"Il est toujours difficile", reconnaît Lavigne Delville (1997: 98), "de traduire une connaissance générale en outils opérationnels". En effet, des concepts considérés comme monnaie courante en sciences sociales - société civile, capital social. transparence, participation - s'avèrent singulièrement difficiles à décortiquer dès lors qu'on s'efforce de les traduire dans le langage des intervenants. Quelles sont les composantes la société civile? Comment mesurer le capital social? Par quelles initiatives promouvoir la transparence, évaluer la participation, encourager la bonne gouvernance? Autant de questions d'une importance capitale et auxquelles les développeurs ne prêtent qu'une attention distraite. Rendre accessible aux développeurs l'outillage conceptuel des sciences sociales nous apparaît comme la condition sine qua non d'un dialogue constructif.

Dans son rôle de trait d'union entre bénéficiaires et bailleurs de fonds le chercheur devrait s'efforcer de faciliter l'interface entre les deux. Ce rôle de facilitateur, nul n'est mieux qualifié pour le jouer que les socio-anthropologues, car "ce sont eux qui connaissent les populations, qui savent leur parler, qui savent les comprendre (...) et peuvent les défendre le cas échéant" (Lavigne Delville 1997 : 100). Rares, cependant, sont ceux qui ont la volonté ou la capacité de jouer ce rôle. Comment communiquer le point de vue des collectivités locales? Comment interpréter les finalités d'un projet auprès des bénéficiaires? Comment traduire la perception villageoise de la décentralisation en termes qui ont du sens pour les paysans et les développeurs? Comment surmonter les blocages de communication entre paysans et développeurs ("Quand je suis devant un bailleur", avoue le président d'une ONG du Burkina, "c'est comme si quelqu'un avait mis une cale à mon vélo !" Peemans et al. $1997: 118)$ ? Sans doute n'est-ce pas l'un des moindres mérites des travaux de Lavigne Delville, Olivier de Sardan et Laurent de s'interroger sur ces questions, et de nous fournir des éléments de réponse. En dernière analyse, cependant, rien ne peut remplacer la connaissance pratique, ce que James Scott, reprenant l'expression de Marcel Détienne et Jean-Pierre Vernant, appelle la "métis" (Scott 1998: 311). Ce savoir-faire, ou plutôt cet "art-de-faire", qui recouvre une large gamme de connaissances acquises sur le terrain, en face d'un champ politique qui ne cesse de se transformer au rythme des réformes, c'est malheureusement ce que trop de développeurs négligent au profit de modèles soigneusement aseptisés qui n'ont qu'un rapport lointain avec la réalité.

Qu'on le veuille ou non, tout projet de développement a des incidences politiques, et ceci est particulièrement vrai des projets de décentralisation. En essayant de naviguer entre le politique et le technique, le chercheur est souvent confronté à des choix 
difficiles. Au niveau de la société civile le choix est entre associations soutenues par le gouvernement et celles qui font figure d'opposantes; dans le domaine de la décentralisation un excès de zèle dans le sens d'une trop grande autonomie des collectivités locales peut facilement provoquer les foudres ministérielles; certaines "circonscriptions" seront incorporées dans un projet et d'autres exclues pour des raisons d'ordre politique. Soyons francs: il n'appartient pas au chercheur de se substituer aux décideurs du pays hôte, ou d'aller à l'encontre des décisions prises par les bailleurs de fonds. Compte-tenu de la marge de manœuvre dont il dispose, son rôle est plutôt de s'interroger sur les conséquences politiques des projets, et ce faisant mettre à jour leurs véritables enjeux. Dans le cas qui nous intéresse plus particulièrement, celui de la décentralisation, il s'agit avant tout de faire la part de ce qui est porteur de promesses pour le monde rural, et de ce qui est supercherie. L'exercice, si j'ai bonne mémoire, n'est pas sans danger...

Revenons enfin à l'éditorial de Cauris (décembre 1994): "Parler de décentralisation aujourd'hui est une gageure parce qu'clic déclenche des passions d'une telle violence qu'on réalise seulement à ce moment les véritables enjeux qu'elle recouvre..." On comprend que ce n'est pas sans inquiétude que j'arrive au terme de cette discussion : puisse mon propos susciter la réflexion plutôt que la violence, le dialogue plutôt que le refus, en bref cette "entente" que recèlent en elles-mêmes les sociétés rurales, et sans laquelle rien de constructif ne peut se faire.

\section{BIBLIOGRAPHIE}

Blundo, G. 1995. "Les courtiers du développement en milieu rural sénégalais". Cahiers d'Etudes Africaines XXXV (137) : 73-101.

Ferguson, J. 1994. The Anti-Politics Machine. "Development", Depoliticization, and Bureaucratic Power in Lesotho. Minneapolis : University Press of Minnesota.

Jacob, J.-P. \& G. Blundo, en collaboration avec N. Bako-Arifari, K. Bprhaug et P.-J. Laurent, Socio-anthropologie de la décentralisation en milieu rural africain. Bibliographie sélective et commentée. Genève : IUED (Itinéraires, série "Notes et Travaux" 49).

Laurent, P.-J. 1995. Les pouvoirs Politiques Locaux et la Décentralisation au Burkina Faso. Louvain-la-Neuve : CIDEP (Cahiers du CIDEP 26).

Lavigne Delville, P. 1997. "A quoi servent les sciences sociales dans les projets de développement? Points de vue d'un «agent double»". Bulletin de l'APAD 14 :79-107.

Mamdani, M. 1996. Citizen and Subject : Contemporary Africa and the Legacy of Late Colonialism. Princeton : Princeton University Press.

Martin, D.-C. (dir.) 1998. Les nouveaux langages du politique en Afrique Orientale. Paris : IFRA Karthala. 
Médard, J.-F. 1982. "The Underdeveloped State in Africa : Political Clientelism or Neo-patrimonialism ?". In Clapham, C. (ed.) Private Patronage and Public Power, New York, St. Martin's Press : 162-183.

Olivier de Sardan, J.-P. 1995. Anthropologie et développement. Essai en socio-anthropologie du développement. Paris : APAD-Karthala.

Peemans, J.-P., Esteves, A. \& P.-J. Laurent 1997. Stabilité politique, ethnicité et dimensions socio-économiques de la gouvernementalité locale. Bruxelles : Centre Interuniversitaire de la Communauté Française de Belgique.

Putnam, R. 1993. Making Democracy Work : Civic Traditions in Modern Italy. Princeton : Princeton University Press.

Scott, J. 1998. Seeing Like a State. New Haven : Yale University Press.

Thompson, I. \& C. Coulibali 1994. "Une simple querelle de mots ?". Cauris (Bamako). décembre $1994: 3$.

\section{AUTEUR}

\section{RENÉ LEMARCHAND}

University of Florida, Center for African Studies, PO Box 115560. Gainesville, FL 32611-5560 (USA). Tél. +2-352-392.21.87 ; Fax.+2-352-392.24.35.- Renelemar@aol.com 